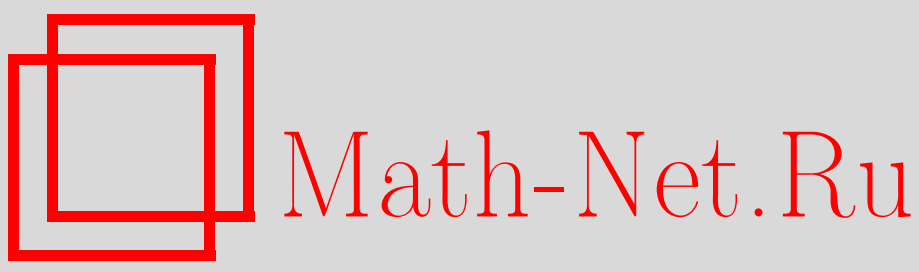

П. В. Морозов, Лиувиллева классификация интегрируемых систем случая Клебша, Матем. сб., 2002, том 193, номер 10, 113-138

DOI: https://doi.org/10.4213/sm687

Использование Общероссийского математического портала Math-Net.Ru подразумевает, что вы прочитали и согласны с пользовательским соглашением http://www.mathnet.ru/rus/agreement

Параметры загрузки:

IP: 54.164 .48 .24

26 апреля 2023 г., 13:07:15 
УДК $515.1+521$

\author{
П.В. Морозов
}

\title{
Лиувиллева классификация интегрируемых систем случая Клебша
}

\begin{abstract}
В работе вычислены инварианты Фоменко-Цишанга для интегрируемого случая Клебша в динамике твердого тела. Таким образом полностью описана структура слоений Лиувилля, возникающих на изоэнергетических поверхностях этой интегрируемой гамильтоновой системы. В качестве следствия установлена лиувиллева эквивалентность случаев Эйлера и Клебша при определенных значениях параметров.

Библиограф̆ия: 24 названия.
\end{abstract}

\section{§1. Введение}

Случай Клебша движения тяжелого твердого тела в жидкости - один из классических случаев интегрируемости в динамике твердого тела. В разное время его исследованием занимались многие выдаюшиеся математики и физики-теоретики. В 1869 году Кирхгофф [1] показал, что вопрос о движении твердого тела в идеальной несжимаемой жидкости сводится к интегрированию системы обыкновенных дифференциальных уравнений с шестью неизвестными, указал три общих интеграла этой системы и нашел четвертый дополнительный интеграл для тела, обладающего осью симметрии. В последующие годы Томсон, Кобб, Ламб, Клебш [2], В.А. Стеклов [3], А.М. Ляпунов [4] и другие ученые посвящали свои работы поиску различњых вариантов дополнительных условий, при которых указанная система допускает четвертый квадратичный интеграл. Новые интересные подходы были разработаны С.А. Чапльгиным [5], [6] и развиты Е. И. Харламовой [7].

Следует отметить, что во всех перечисленных работах авторы так или иначе стремились аналитическим путем изучить движение тела. Зачастую найденные явные формулы не давали качественного представления о характере движения твердого тела в силу своей крайней сложности.

Работы М.П. Харламова, Т.И. Погосяна и А.А. Ошемкова по исследованию случая Клебша связаны уже с применением новых топологических методов при исследовании геометрии фазового пространства системы. М.П. Харламовым [8], [9] был подробно исследован случай нулевой постоянной площадей. Т.И. Погосян [10], [11] построил бифуркационные диаграммы в общем случае, детально изучил топологию особых совместных уровней интегралов. В 80-х годах А.Т. Фоменко и его школой была создана теория лиувиллевой классификации интегрируемых гамильтоновых систем с двумя степенями свободы [12], [13], что дало мощный толчок для продолжения качественных исследований, связанных с задачей о движении твердого тела. В частности, А. А. Ошемковым [14], [15] была установлена боттовость дополнительного интеграла Клебша на почти всех изоэнергетических по-

(C) П. в. Морозов 2002 
верхностях, указаны типы особенностей бифуркационной диаграммы, а также вычислены грубые лиувиллевы инварианты слоения фазового пространства. В настоящей работе получен новый результат: вычислены все тонкие лиувиллевы инварианты (инварианты Фоменко-Цишанга) систем случая Клебша. Таким образом целиком описана топология лиувиллевых слоений, возникающих на трехмерных изоэнергетических поверхностях фазового пространства системы. Это позволило, в частности, установить лиувиллеву эквивалентность случаев Клебша и Эйлера при определенных значениях параметров (см. теорему 3 ).

\section{§2. Постановка задачи}

Хотя результаты самого А. Клебша [2] относятся к движению тела в идеальной несжимаемой жидкости, мы будем отталкиваться от другой физической интерпретации. Подробное описание и исследование физической задачи, которую рассматривал Клебш, можно найти в книге Ламба [16]. Мы же будем рассматривать задачу о движении твердого тела вокруг неподвижного центра масс в линейном поле сил, как это сделано в работах [8]-[11], [17]. Такой подход позволяет усмотреть тесную связь между случаем Клебша и случаем Эйлера, играюшим фундаментальную роль в динамике твердого тела. Несложно убедиться, что обе физические задачи приводят к эквивалентньм уравнениям.

Рассмотрим несимметричное твердое тело, закрепленное в центремасс $O$. Пусть на каждую частицу тела действует сила

$$
\delta \vec{F}=-\gamma \frac{\vec{r}}{r^{3}} \delta m
$$

где $\gamma$ - постоянная, а $\vec{r}$ - вектор, проведенный из некоторой фиксированной в пространстве точки $P$ в частицу $\delta m$. При $\gamma>0$ мы имеем задачу о притяжении твердого тела материальной точкой $P$, а при $\gamma<0$ - задачу об отталкивании.

Свяжем с телом подвижную систему координат, в качестве осей которой выберем главные центральные оси инерции твердого тела. Пусть

$$
A_{1}<A_{2}<A_{3}
$$

- главные центральные моменты инерции тела.

Введем единичный вектор

$$
\vec{\nu}=\frac{\overrightarrow{O P}}{|O P|},
$$

и пусть $\nu_{1}, \nu_{2}, \nu_{3}$ - его компоненты в подвижных осях.

Будем считать величину $d=|O P|$ достаточно большой по сравнению с размерами тела. Тогда, сохраняя в разложении момента внешних сил относительно неподвижной точки лишш члены до порядка $d^{-3}$, запишем его проекции на подвижные оси:

$$
M_{1}=3 \frac{\gamma}{d^{3}}\left(A_{3}-A_{2}\right) \nu_{2} \nu_{3}, \quad M_{2}=3 \frac{\gamma}{d^{3}}\left(A_{1}-A_{3}\right) \nu_{3} \nu_{1}, \quad M_{1}=3 \frac{\gamma}{d^{3}}\left(A_{2}-A_{1}\right) \nu_{1} \nu_{2} .
$$

Полученные выражения являются общепринятьм приближением центрального ньютоновского поля сил и представляют собой в точности моменты силового поля, 
параллельного вектору $\overrightarrow{O P}$ и вдоль его направления меняющегося по линейному закону.

Положим $\varepsilon=3 \gamma / d^{3}$. Случай Эйлера получается из случая Клебша при условии постоянства силы вдоль силовых линий, что соответствует удалению точки $P$ на бесконечность. Точнее, надо осушествить следующий предельный переход:

$$
\frac{\gamma}{d^{2}} \rightarrow C, \quad \varepsilon=\frac{3 \gamma}{d^{3}} \rightarrow 0 \quad \text { при } \quad d \rightarrow \infty
$$

Уравнения Эйлера-Пуассона в рассматриваемой задаче таковы:

$$
\begin{gathered}
A_{1} \frac{d \omega_{1}}{d t}=\left(A_{2}-A_{3}\right)\left(\omega_{2} \omega_{3}-\varepsilon \nu_{2} \nu_{3}\right) \\
A_{2} \frac{d \omega_{2}}{d t}=\left(A_{3}-A_{1}\right)\left(\omega_{1} \omega_{3}-\varepsilon \nu_{3} \nu_{1}\right) \\
A_{3} \frac{d \omega_{3}}{d t}=\left(A_{1}-A_{2}\right)\left(\omega_{1} \omega_{2}-\varepsilon \nu_{1} \nu_{2}\right) \\
\frac{d \nu_{1}}{d t}=\omega_{3} \nu_{2}-\omega_{2} \nu_{3} \\
\frac{d \nu_{2}}{d t}=\omega_{1} \nu_{3}-\omega_{3} \nu_{1} \\
\frac{d \nu_{3}}{d t}=\omega_{2} \nu_{1}-\omega_{1} \nu_{2}
\end{gathered}
$$

здесь $\omega_{1}, \omega_{2}, \omega_{3}-$ проекции угловой скорости тела на подвижные оси.

Система обладает следующими первыми интегралами:

$$
\begin{gathered}
\nu_{1}^{2}+\nu_{2}^{2}+\nu_{3}^{2}=1-\text { геометрический интеграл, } \\
J=A_{1} \omega_{1} \nu_{1}+A_{2} \omega_{2} \nu_{2}+A_{3} \omega_{3} \nu_{3}-\text { интеграл площадей, } \\
H=\frac{1}{2}\left(A_{1} \omega_{1}^{2}+A_{2} \omega_{2}^{2}+A_{3} \omega_{3}^{2}\right)+\frac{\varepsilon}{2}\left(A_{1} \nu_{1}^{2}+A_{2} \nu_{2}^{2}+A_{3} \nu_{3}^{2}\right)-\text { интеграл энергии, } \\
K=\frac{1}{2}\left(A_{1}^{2} \omega_{1}^{2}+A_{2}^{2} \omega_{2}^{2}+A_{3}^{2} \omega_{3}^{2}\right)-\frac{\varepsilon}{2}\left(A_{2} A_{3} \nu_{1}^{2}+A_{3} A_{1} \nu_{2}^{2}+A_{1} A_{2} \nu_{3}^{2}\right)-\text { интеграл Клебша. }
\end{gathered}
$$

Рассмотрим алгебру Ли $е(3)$ группы Ли $E(3)$ движений трехмерного евклидова пространства (см. [18], [19]). На линейном пространстве $e(3)^{*}$ определена скобка Ли-Пуассона двух произвольных гладких функций $f$ и $g$ :

$$
\{f, g\}(x)=x\left(\left[d_{x} f, d_{x} g\right]\right),
$$

где $x \in e(3)^{*}, \mathrm{a}[\cdot, \cdot]$ - коммутатор в алгебре Ли $e(3)$.

В естественных координатах

$$
S_{1}, S_{2}, S_{3}, R_{1}, R_{2}, R_{3}
$$

на пространстве $e(3)^{*}$ эта скобка записьвается следующим образом:

$$
\left\{S_{i}, S_{j}\right\}=\varepsilon_{i j k} S_{k}, \quad\left\{R_{i}, S_{j}\right\}=\varepsilon_{i j k} R_{k}, \quad\left\{R_{i}, R_{j}\right\}=0
$$


где

$$
\{i, j, k\}=\{1,2,3\}, \quad \varepsilon_{i j k}=\frac{1}{2}(i-j)(j-k)(k-i) .
$$

Введем обозначения: $A=\operatorname{diag}\left(A_{1}, A_{2}, A_{3}\right), S=\left(S_{1}, S_{2}, S_{3}\right), R=\left(R_{1}, R_{2}, R_{3}\right)$, тогда после замены $S=-A \omega, R=\nu$ рассматриваемые уравнения перепишутся в виде обобщенных уравнений Кирхгофа:

$$
\begin{gathered}
\dot{S}=\frac{\partial H}{\partial S} \times S+\frac{\partial H}{\partial R} \times R \\
\dot{R}=\frac{\partial H}{\partial S} \times R
\end{gathered}
$$

которые, в свою очередь, при помощи введенной скобки Ли-Пуассона можно переписать в виде гамильтоновой системы на $e(3)^{*}$ :

$$
\dot{S}_{i}=\left\{S_{i}, H\right\}, \quad \dot{R}_{i}=\left\{R_{i}, H\right\}
$$

При этом первые два интеграла системы перейдут в функции Казимира

$$
f_{1}=R_{1}^{2}+R_{2}^{2}+R_{3}^{2}, \quad f_{2}=S_{1} R_{1}+S_{2} R_{2}+S_{3} R_{3}
$$

а гамильтониан $H$ и дополнительньй интеграл Клебша $K$ запишутся в виде

$$
\begin{gathered}
H=\frac{S_{1}^{2}}{2 A_{1}}+\frac{S_{2}^{2}}{2 A_{2}}+\frac{S_{3}^{2}}{2 A_{3}}+\frac{\varepsilon}{2}\left(A_{1} R_{1}^{2}+A_{2} R_{2}^{2}+A_{3} R_{3}^{2}\right), \\
K=\frac{1}{2}\left(S_{1}^{2}+S_{2}^{2}+S_{3}^{2}\right)-\frac{\varepsilon}{2}\left(A_{2} A_{3} R_{1}^{2}+A_{1} A_{3} R_{2}^{2}+A_{1} A_{2} R_{3}^{2}\right) .
\end{gathered}
$$

Заменой $A_{i}^{\prime}=\sqrt{|\varepsilon|} A_{i}$ и делением гамильтониана на $\sqrt{|\varepsilon|}$ добиваются того, что $\varepsilon= \pm 1$. Причем положительный $\varepsilon$ соответствует случаю притяжения, а отрицательный - случаю отталкивания.

Оказывается, что система является невырожденной гамильтоновой на совместных четырехмерных поверхностях уровня функций $f_{1}$ и $f_{2}$ (см. [18], [20]):

$$
M_{c, g}^{4}=\left\{f_{1}=R_{1}^{2}+R_{2}^{2}+R_{3}^{2}=c, f_{2}=S_{1} R_{1}+S_{2} R_{2}+S_{3} R_{3}=g\right\} .
$$

Для почти всех значений $c$ и $g$ эти поверхности являются неособыми гладкими подмногообразиями в $e(3)^{*}$, а при $c>0$ диффеоморфны $T S^{2}$. В дальнейшем будем считать, что $c$ и $g$ являются именно такими регулярными значениями. Симплектическая структура задается на $M_{c, g}^{4}$ ограничением скобки Ли-Пуассона из объемлющего пространства $e(3)^{*}$. Поскольку линейное преобразование $S^{\prime}=S$, $R^{\prime}=\mu R$, где $\mu=\mathrm{const}$, coхраняет скобку, будем далее всегда считать, что $c=1$.

Отметим одно важное обстоятельство. Несложно убедиться, что в случае Клебша любые варианты $H$ и $K$ могут быть представлены в виде линейной комбинации двух коммутирующих функций вида:

$$
\begin{gathered}
H_{0}=\left(S_{1}^{2}+S_{2}^{2}+S_{3}^{2}\right)+\left(c_{1} R_{1}^{2}+c_{2} R_{2}^{2}+c_{3} R_{3}^{2}\right) \\
K_{0}=\left(c_{1} S_{1}^{2}+c_{2} S_{2}^{2}+c_{3} S_{3}^{2}\right)-\left(c_{1}^{2} R_{1}^{2}+c_{2}^{2} R_{2}^{2}+c_{3}^{2} R_{3}^{2}\right)
\end{gathered}
$$


где $c_{1}+c_{2}+c_{3}=0$. Таким образом, мы фактически понижаем число параметров до двух.

Поясним вкратце, в чем состоит цель нашей работы. Рассмотрим интегрируемую систему с двумя степенями свободы на $M^{4}$ с гамильтонианом $H$ и дополнительным интегралом $K$. Зафиксировав значение гамильтониана $H=h$, мы получаем в $M^{4}$ изоэнергетическую поверхность $Q_{h}^{3}$, на которой возникает слоение Лиувилля. Две гамильтоновы системы на $Q_{h}^{3}$ и $Q_{h}^{\prime 3}$ называются лиувиллево әквивалентными, если между этими поверхностями существует гомеоморфизм, сохраняющий это слоение. Известно [12], [13], что при боттовости ограничения дополнительного интеграла необходимым и достаточньм условием лиувиллевой эквивалентности двух систем является изоморфизм их меченых молекул. Меченая молекула (или инвариант Фоменко-Цишанга) представляет собой граф̆ с числовыми метками на ребрах и с вершинами особого вида - атомами. Граф с указанньми типами атомов несет в себе информацию о последовательности бифуркаций торов в слоении Лиувилля на трехмерной поверхности. Числовые метки на ребрах молекулы показывают, каким образом окрестности особых поверхностей уровня интегралов, отвечающих этим бифуркациям, склеены между собой. Подробное изложение теории инвариантов Фоменко-Цишанга можно найти в [12], [13].

В настоящей работе вычислены все меченые молекулы, соответствующие слоениям Лиувилля, возникаюшим на изоэнергетических поверхностях в случае Клебша (см. теорему 2).

Стоит отметить, что инварианты Фоменко-Цишанга уже вычислены разными авторами для большинства случаев интегрируемости твердого тела (см. [12; т. 2, гл. 5]). За это время выработались определенная техника и обозначения. Так, мы будем постоянно ссылаться на работу А.В. Болсинова, П. Рихтера, А.Т. Фоменко [21], в которой полностью вычислены инварианты Фоменко-Цишанга для случая Ковалевской и классифицированы все соответствующие слоения Лиувилля.

\section{§ 3. Бифуркационные диаграммы систем случая Клебша}

Рассмотрим отображение момента

$$
H_{0} \times K_{0}: M_{g}^{4} \rightarrow \mathbb{R}^{2}(h, k),
$$

здесь индекс $g$ - соответствующее значение постоянной площадей.

Далее в этом параграфе мы вкратце приводим результаты, полученные А. А. Ошемковым [14], [15] и Т. И. Погосяном [8], [10], [11].

Бифуркационная диаграмма сушественно зависит от параметра $g$ - постоянной площадей. При противоположных значениях параметра диаграммы одинаковы, поэтому далее везде считаем $g \geqslant 0$. Имеется три принципиально различных случая:

1) $0<g^{2}<p_{1}$ (см. рис. 1a)), $g=0$ (рис. 1b)),

2) $p_{1}<g^{2}<p_{2}$ (рис. 1c)),

3) $g^{2}>p_{2}($ рис. 1d)),

здесь $p_{1}, p_{2}$ - некоторые константы, зависящие лишш от $c_{1}, c_{2}, c_{3}$.

Дуги бифуркационной диаграммы - это множество точек, в которых ранг отображения момента равен единище. Будем обозначать их малыми греческими буквами. Дуги $\alpha_{2}\left(\alpha_{3}\right), \gamma_{2}\left(\gamma_{1}\right)$ и $\beta$ при $h \rightarrow \infty$ имеют асимптоты $k=c_{3} h+c_{1} c_{2}$, 


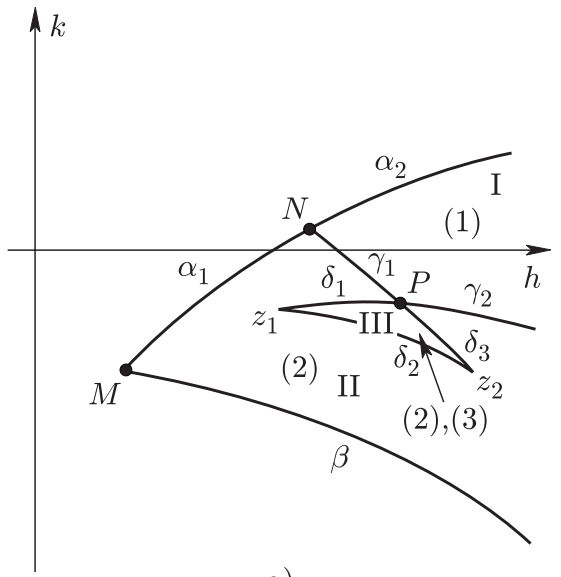

a)

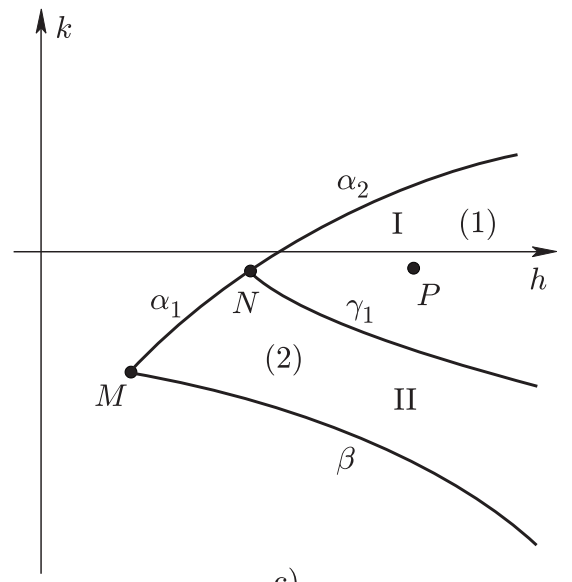

c)

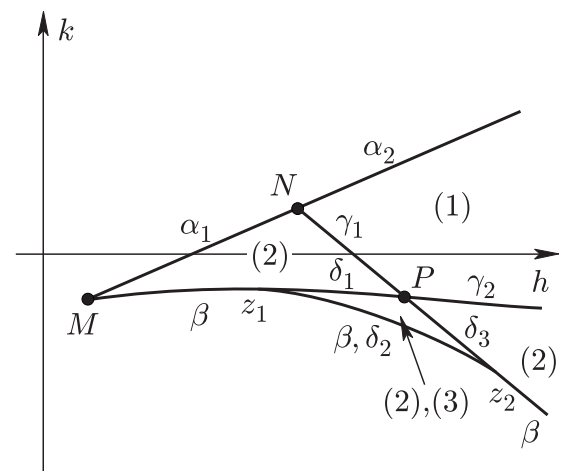

b)

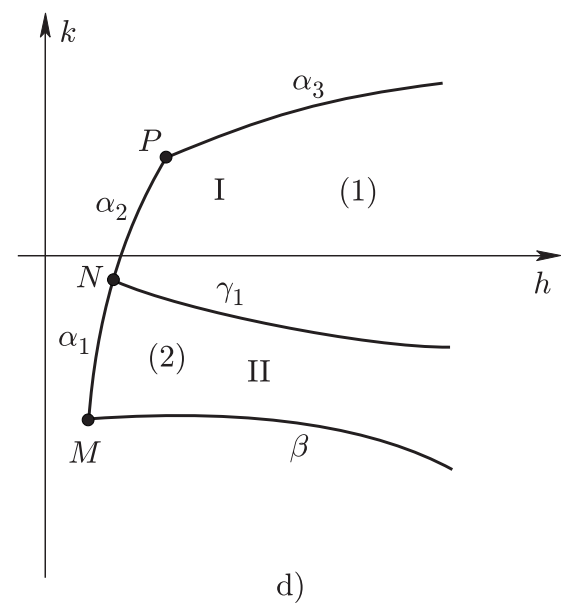

Рис. 1.

$k=c_{2} h+c_{1} c_{3}$ и $k=c_{1} h+c_{2} c_{3}$ соответственно. Дугам диаграммы соответствуют бифуркации следующих типов:

\begin{tabular}{|c|c|}
\hline Дуга & Тип бифуркации \\
\hline$\alpha_{1}, \alpha_{2}, \alpha_{3}, \beta, \delta_{2}$ & $A+A$ \\
\hline$\delta_{1}, \delta_{3}$ & $B+B$ \\
\hline$\gamma_{1}, \gamma_{2}$ & $C_{2}$ \\
\hline
\end{tabular}

Через $M, N, P$ обозначены точки, в которых ранг отображения момента падает до нуля. При $g^{2} \neq p_{1}, p_{2}$ они представляют собой так называемые невырожденные положения равновесия. Эти точки имеют координаты $\left(g^{2}+c_{i}, c_{i}\left(g^{2}-c_{i}\right)\right)$, $i=1,2,3$, соответственно. Точки возврата $z_{1}, z_{2}$ относятся к вырожденным особенностям отображения момента. Типы особенностей этих пяти точек таковы 
(подробнее см. [12; т. 1, гл. 9 и т. 2, гл. 1]):

\begin{tabular}{|c|c|}
\hline Точка & Тип \\
\hline$M, P$ (при $\left.g^{2}>p_{2}\right)$ & центр-центр $A \times A$ \\
\hline$N$ & седло-центр $A \times C_{2}$ \\
\hline$P$ (при $\left.g^{2}<p_{1}\right)$ & седло-седло $\left(C_{2} \times C_{2}\right) / \mathbb{Z}_{2}$ \\
\hline$P$ (при $\left.p_{1}<g^{2}<p_{2}\right)$ & фокус-фокус \\
\hline$z_{1}, z_{2}$ (при $\left.g=0\right)$ & эллиптическая вилка (рitchfork) \\
\hline$z_{1}, z_{2}$ (при $\left.g \neq 0\right)$ & бифуркация седло-узел \\
\hline
\end{tabular}

Области между дугами бифуркационной диаграммы (будем называть их камерами) заполняют регулярные точки отображения момента. Прообразами этих точек по теореме Лиувилля является несвязное объединение некоторого числа двумерных торов. Над камерой I (см. рис. 1) “висят” два одинаковых тора. Над камерой II также висят два одинаковых тора. В камере III картина более сложная: в каждую ее точку попадают сразу четыре тора, которые можно разбить на две пары. Два из них проникают в камеру III из камеры II, “проскальзывая" сквозь дугу $\delta_{2}$. Два оставшихся тора камеры III при переходе через эту дугу испытывают $A$-бифуркацию и исчезают.

Как видно, прообразами одной регулярной точки бифуркационной диаграммы могут служить принципиально разные торы Лиувилля в том смысле, что они испытывают разные топологические перестройки при гладкой изотопии в $M^{4}$. Более того, в одну точку бифуркационной диаграммы могут одновременно попасть регулярные торы Лиувилля и особые поверхности уровня интегралов, как это происходит на дуге $\delta_{2}$. В связи с этим стоит отметить, что бифуркационную диаграмму естественно рассматривать как проекцию на плоскость $\mathbb{R}^{2}(H, K)$ двумерного комплекса - базы слоения Лиувилля. Тогда можно говорить о двулистном накрытии над камерами I и II и о четырехлистном накрытии над камерой III, где каждый лист накрытия - это в точности двумерная клетка этого комплекса.

Для дальнейшего нам будет удобно разбить торы на семейства. В силу вьшесказанного это естественно сделать следующим образом:

- к семейству (1) отнесем два идентичных тора над камерой I;

- семейство (2) - торы камеры II и два тора камеры III, “проникающие” туда сквозь дугу $\delta_{2}$;

- семейство (3) - два оставшихся тора камеры III.

Заметим, что семейство отображений момента $M_{g}^{4} \rightarrow \mathbb{R}^{2}$ можно рассматривать как одно отображение пятимерного многообразия $S^{2} \times \mathbb{R}^{3} \rightarrow \mathbb{R}^{3}(g, h, k)$. Наши двумерные диаграммы являются плоскими сечениями обшей трехмерной. При движении плоскости сечения вдоль $g$ наблюдается следуюшая картина. Когда $g$ отделяется от нуля, $\delta_{2}$-дуга соскальзьвает с $\beta$-дуги. Далее она вместе с остальньми $\delta$-дугами начинает стягиваться к точке $P$, а излом между двумя $\gamma$-дугами начинает сглаживаться. При $g^{2}=c_{1}$ этот процесс завершается: $\delta$-дуги исчезают в точке $P$, а $\gamma$-дуги выпрямляются в одну гладкую кривую. Далее точка $P$ соскальзывает с $\gamma$-дуги, превращаясь в особенность типа фокус-фокус. При $g^{2}=c_{2}$ она оказывается на дуге $\alpha_{2}$. При дальнейшем увеличении $g$ точка $P$ преврашается в особенность типа центр-центр и начинает выдавливать излом в $\alpha$-дуге.

Пусть гамильтониан $H$ представим в виде $H=\alpha H_{0}+\beta K_{0}$. Тогда при отображении момента изоэнергетические поверхности будут отображаться в сечения 
бифуркационной диаграммы семейством параллельных прямых $\alpha h+\beta k=c$. При этом случаю притяжения соответствуют прямые из зоны 1 (см. рис. 2), а случаю отталкивания - прямые из зоны 2 . Будем рассматривать только те из них, которые не проходят через особые точки бифуркационной диаграммы и пересекают ее дуги трансверсально. На рис. 3 каждой прямой (с точностью до гладкой изотопии) присвоен номер. Часть из них для удобства изображена кривыми, которые соответствуют изотопным им прямым при других значениях параметров $c_{1}, c_{2}$ и $c_{3}$.

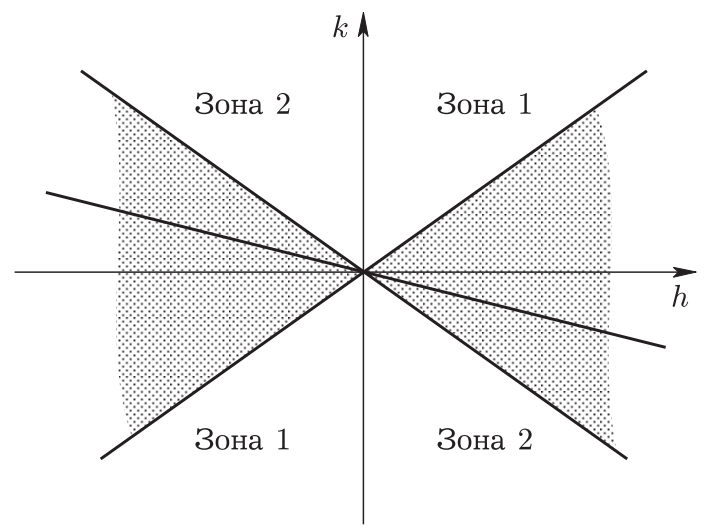

Рис. 2.

Вычислив меченую молекулу для каждой такой прямой, мы опишем все топологически устойчивые слоения Лиувилля на изоэнергетических поверхностях. Однако мы решим более обшую задачу: научимся вычислять меченую молекулу для произвольной допустимой кривой. Под допустимой кривой понимается гладкая кривая, пересекаюшая дуги бифуркационной диаграммы трансверсально и не проходящая через ее особые точки. Потребуем также, чтобы кривая пересекала образ отображения момента по компактному множеству. Для обоснования корректности поставленной задачи необходимо отметить, что ограничение системы на 3-подмногообразие, лежашее в прообразе допустимой кривой, будет боттовским (А. А. Ошемков [14], [15]).

\section{§4. Классификация "круговых" слоений Лиувилля в случае Клебша}

В случае Клебша бифуркационная диаграмма имеет пять особых точек. Рассмотрим какую-то одну из них. Опишем малую окружность вокруг этой особенности. В ее прообразе имеется некоторое трехмерное многообразие, на котором возникает слоение Лиувилля. Инвариант Фоменко-Цишанга этого слоения называют круговой молекулой данной особенности. Оказывается, что, с одной стороны, круговые молекулы много “знают" о глобальном слоении системы, а с другой - их несложно вычислить.

ТЕОРема 1. В случае Клебиа обнаруживается ровно шесть различных круговых молекул. Их полный список приведен таблище 1.

ДокаЗАтельство. Точка $M$ и точка $P$ при $g^{2}>p_{2}$ имеют тип центр-центр. В $[12 ;$ т. 1, гл. 9$]$ доказано, что в этом случае круговая молекула особенности имеет 

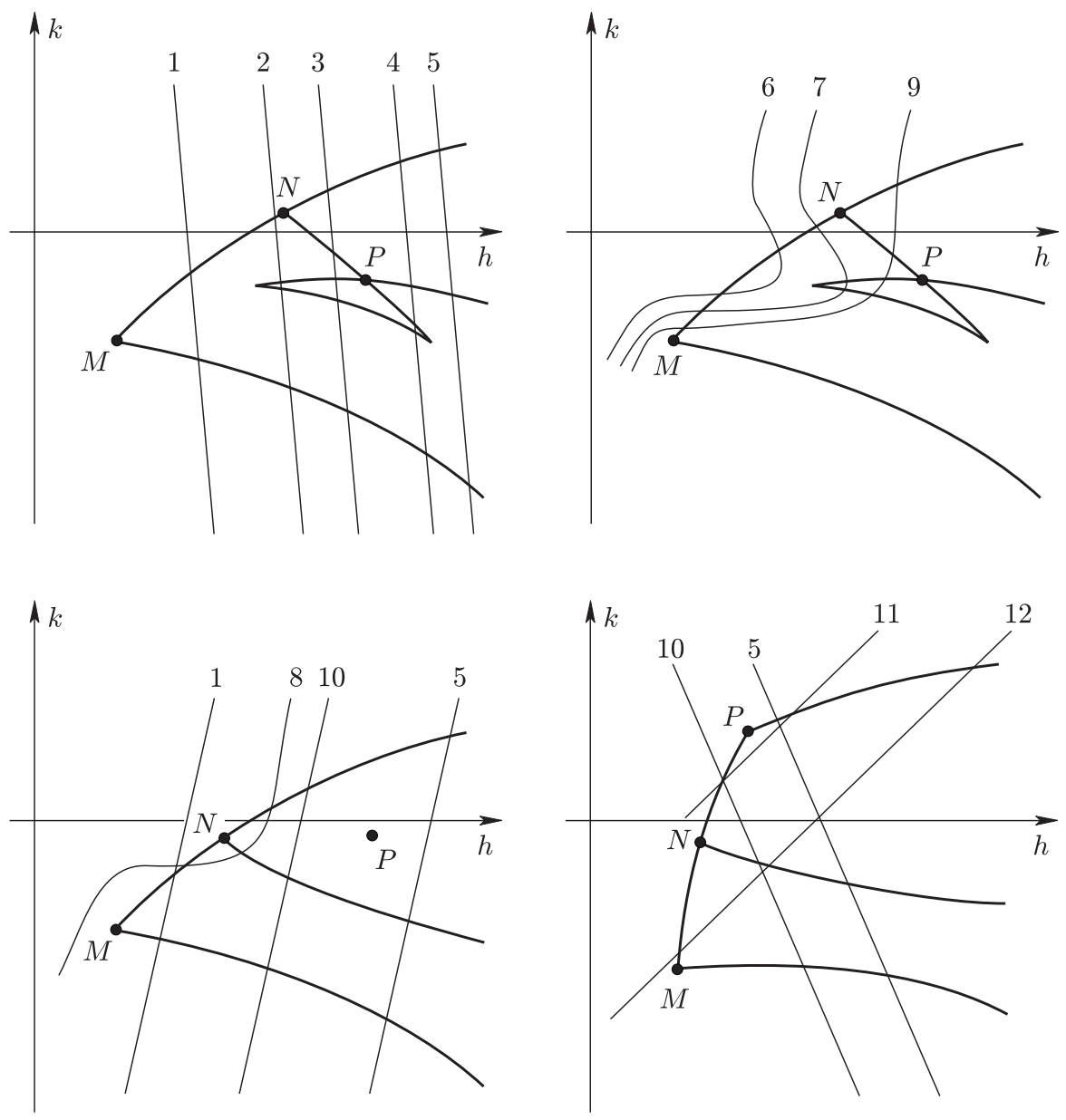

Рис. 3.

вид, указанный для этих точек в таблице 1. Там же доказывается, что в случае особой точки типа седло-центр (точка $N$ ) круговая молекула точки однозначно определяется по ее представлению в виде прямого произведения 2-атомов (в нашем случае $\left.A \times C_{2}\right)$, и указан конкретный вид этой молекулы.

Вырожденные особенности отображения момента интегрируемых гамильтоновых систем с двумя степенями свободы подробно исследованы в работах В.В. Калашникова (мл.) [22], [23] (см. также [12], [21]). Им также были вычислены круговые молекулы наиболее типичных вырожденных особенностей, в том числе и для бифуркаций типа седло-узел и эллиптическая вилка, к которым относятся точки $z_{1}$ и $z_{2}$.

При $g^{2}<p_{1}$ точка $P$ имеет особенность типа седло-седло. Ее круговая молекула указана в [12; т. 2, таблица 1.1(b)]. В [12; т. 1, таблица 9.1], где приведен полный список особенностей типа седло-седло малой сложности, она стоит под номером 17.

Для особенности типа фокус-фокус (точка $P$ при $p_{1}<g^{2}<p_{2}$ ) инвариантом 
ТАБлицА 1. Круговые молекулы

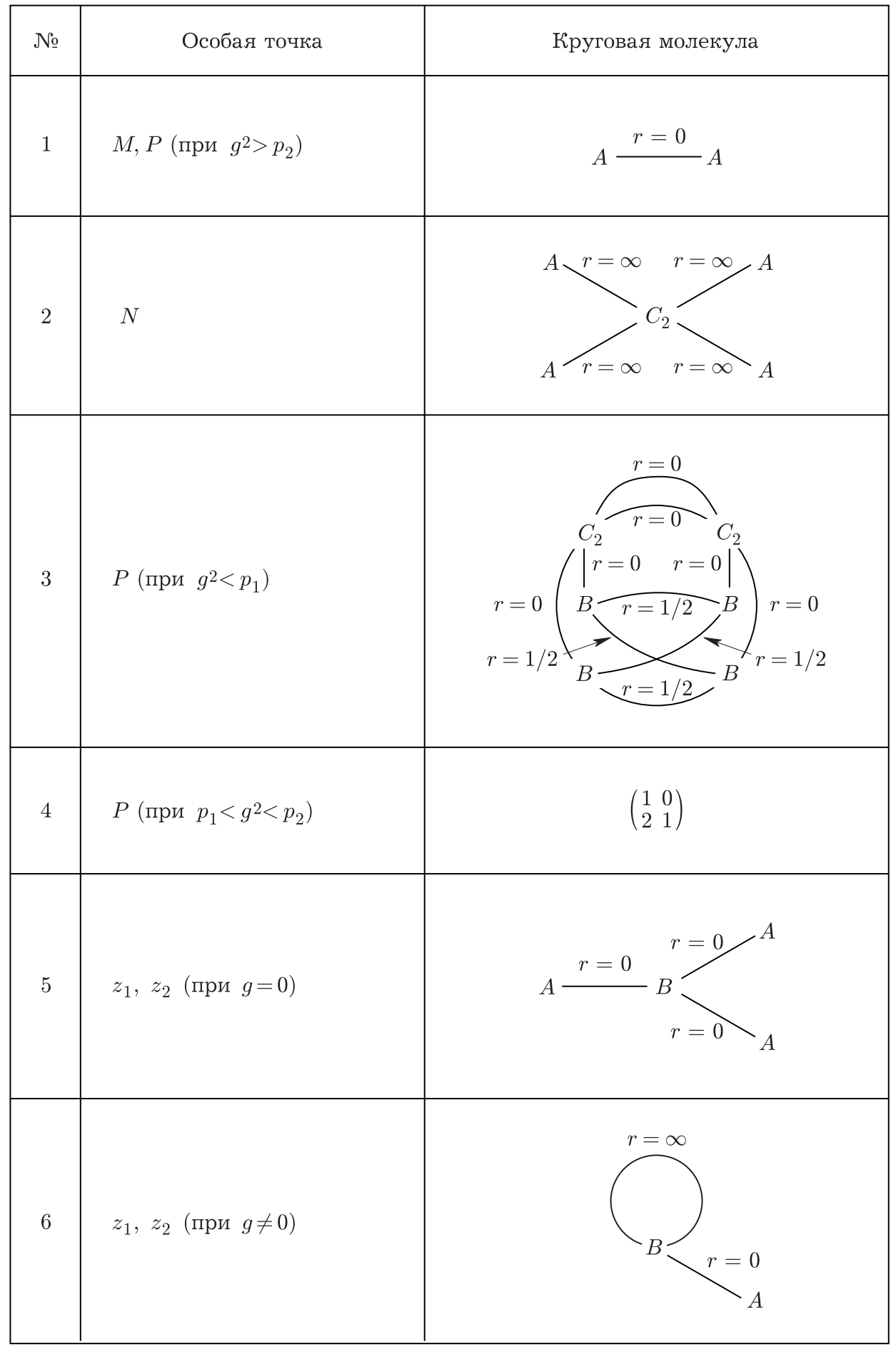


слоения является матрица монодромии. В данном случае она такова: $\left(\begin{array}{ll}1 & 0 \\ 2 & 1\end{array}\right)$. Этот факт указан в [12; т. 2, таблица $1.1(\mathrm{a})]$.

Тем самьм, для каждой особой точки бифуркационной диаграммы мы указали ее круговую молекулу. Теорема доказана.

Стоит отметить, что особенности точек $M, N, P$ (при $\left.g^{2}>p_{2}\right), z_{1}$ и $z_{2}$ являются типичными и встречаются во многих других случаях интегрируемости, в то время как особенности фокус-фокус и седло-седло точки $P$ с указанными круговыми молекулами на данный момент нигде кроме случая Клебша и четырехмерного твердого тела не обнаружены.

\section{§5. Построение допустимых систем координат}

Рассмотрим малый отрезок, трансверсально пересекающий дугу бифуркационной диаграммы. В его прообразе лежит многообразие с краем - 3-атом, край которого состоит из некоторого числа (в зависимости от типа бифуркации) граничных торов. Нам предстоит выбрать на каждом таком торе базис в его фундаментальной группе. Для того чтобы система координат, определяемая этим базисом, была допустимой, она должна удовлетворять ряду формальных требований (см. [12; т. 1]). Оказывается, что бифуркация однозначно определяет на граничных торах некоторый цикл (в случае атома $A$ он определен с точностью до ориентации). Будем обозначать его через $\lambda_{*}$ для бифуркации $*$. В допустимой системе координат он всегда становится первым элементом базиса. В качестве второго элемента берут любой другой цикл, дополняющий первый до базиса. В случае седловых атомов без звездочек, к которым относятся $B$ и $C_{2}$, также требуют, чтобы в совокупности вторые циклы базисов образовывали на 3-атоме глобальное сечение, над которым атом представим как тривиальное $S^{1}$-расслоение.

Дополнительно к этому требуется согласованность ориентаций базисов, которая заключается в следуюшем.

Рассмотрим допустимую кривую. В ее прообразе лежит компактная ориентируемая поверхность $X^{3}$. Зафиксируем на ней какую-либо ориентацию. Выберем произвольный интеграл $f$ на $M^{4}$, монотонный на кривой и коммутирующий с гамильтонианом $H_{0}$. Тогда граничные торы всех атомов можно разделить на положительные и отрицательные (по возрастанию $f$ ). Потребуем, чтобы тройка $\left(\lambda_{1}, \lambda_{2}, \operatorname{grad} f\right)$, где $\lambda_{1}, \lambda_{2}-$ соответственно первый и второй элементы базиса на торе, была положительно ориентирована в $X^{3}$, если тор относится к положительной границе атома, и отрицательно ориентирована в противном случае.

Отметим, что, вообще говоря, меченая молекула зависит от выбора ориентации на $X^{3}$, которую мы фиксировали произвольньм образом. В результате чего в ответе возникают две молекулы для каждой поверхности. Они, разумеется, тесно связаны. Правила перестроек молекул при смене ориентации приведены в [12; гл. 4, т. 1]. Такие молекулы в рамках поставленной задачи различать не принято, так как это не влияет на существование гомеоморфизма, сохраняющего лиувиллево слоение.

Поставим нашей целью предъявить на каждом граничном торе 3-атома допустимую систему координат, выраженную в однозначно определенных базисных циклах самой этой бифуркации и ее ближайших соседей. Для определенности все ато- 


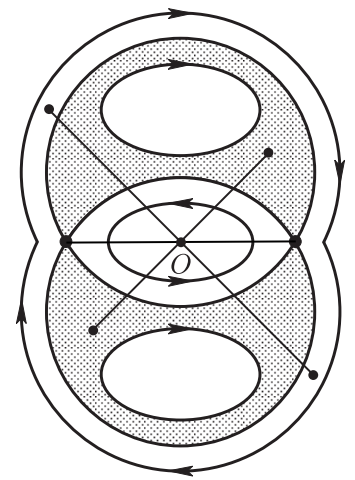

Действие группы $\mathbb{Z}_{2}$

a)

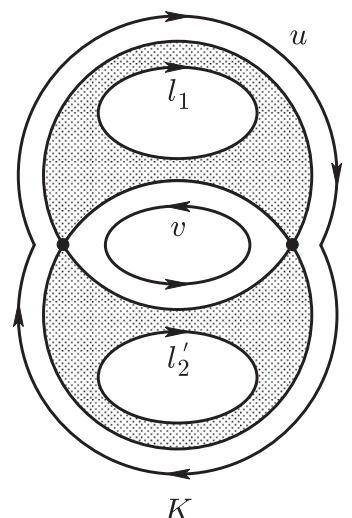

K

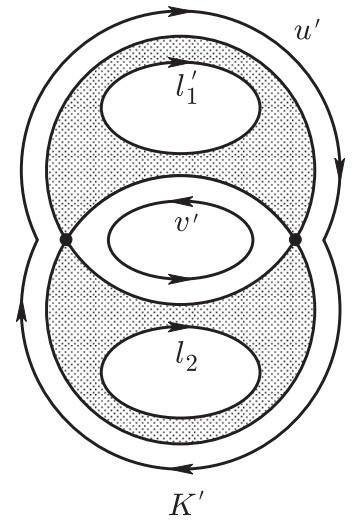

b)
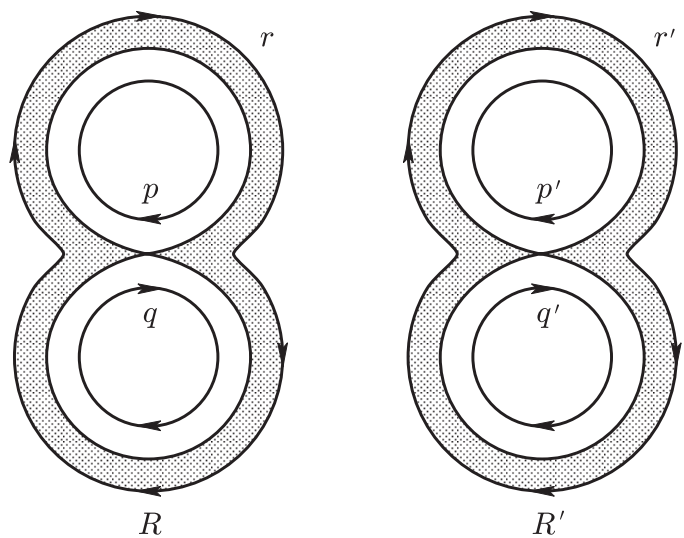

c)

Рис. 4.

мы будем ориентировать по возрастанию $K_{0}$.

Рассмотрим точку $P$ при $g^{2}<p_{1}$. Она имеет тип седло-седло. Из таблицы 9.1 в [12] узнаем, что ее 4-окрестность представима в виде полупрямого произведения $\left(C_{2} \times C_{2}\right) / \mathbb{Z}_{2}$. Там также описано действие группы $\mathbb{Z}_{2}$ на сомножителях, см. рис. 4a). Обозначим 3-атомы, близкие к этой особенности, соответственно через $Q_{\gamma_{1}}, Q_{\gamma_{2}}, Q_{\delta_{1}}, Q_{\delta_{3}}$. На рис. $\left.4 \mathrm{~b}\right)$ изображены два экземпляра атома $C_{2}$, которые перемножаются в прямом произведении: $K$ и $K^{\prime}$. Обозначим граничные циклы на них через $l_{1}, l_{2}, u$ и $v$, как показано на рис. $\left.4 \mathrm{~b}\right)$. Тогда имеем:

1) $Q_{\gamma_{1}}=\left(\left(K \times l_{1}^{\prime}\right) \cup\left(K \times l_{2}^{\prime}\right)\right) / \mathbb{Z}_{2}$;

2) $Q_{\gamma_{2}}=\left(\left(K^{\prime} \times l_{1}\right) \cup\left(K^{\prime} \times l_{2}\right)\right) / \mathbb{Z}_{2}$;

3) $Q_{\delta_{3}}=\left(\left(K \times u^{\prime}\right) \cup\left(K \times v^{\prime}\right)\right) / \mathbb{Z}_{2}$;

4) $Q_{\delta_{1}}=\left(\left(K^{\prime} \times u\right) \cup\left(K^{\prime} \times v\right)\right) / \mathbb{Z}_{2}$.

Заметим, что $l_{1}$ под действием $\mathbb{Z}_{2}$ переходит в $l_{2}$. С учетом этого имеем: 


$$
\begin{gathered}
Q_{\gamma_{1}}=\left(\left(K \times l_{1}^{\prime}\right) \cup\left(K \times l_{2}^{\prime}\right)\right) / \mathbb{Z}_{2}=K \times l_{1}^{\prime} \quad\left(\text { или } K \times l_{2}^{\prime}\right), \\
Q_{\gamma_{2}}=K^{\prime} \times l_{1} \quad\left(\text { или } K^{\prime} \times l_{2}\right), \\
Q_{\delta_{3}}=\left(\left(K \times u^{\prime}\right) \cup\left(K \times v^{\prime}\right)\right) / \mathbb{Z}_{2}=\left(K \times u^{\prime}\right) / \mathbb{Z}_{2} \cup\left(K \times v^{\prime}\right) / \mathbb{Z}_{2} \\
=\left(K / \mathbb{Z}_{2} \times u^{\prime}\right) \cup\left(K / \mathbb{Z}_{2} \times v^{\prime}\right)=\left(R \times u^{\prime}\right) \cup\left(R \times v^{\prime}\right), \\
Q_{\delta_{1}}=\left(R^{\prime} \times u\right) \cup\left(R^{\prime} \times v\right),
\end{gathered}
$$

где $R=K / \mathbb{Z}_{2}=C_{2} / \mathbb{Z}_{2}=B$. Граничные циклы, появившиеся в результате факторизации поверхности $R$, обозначим через $p, q$ и $r$ (см. рис. $4 c))$. Нам необходимо выяснить, как они связаны с $l_{1}, l_{2}, u$ и $v$. Эта нетривиальная часть работы уже проделана: точно такой же случай встречается в работе [21] по системе Ковалевской. Нам остается выписать ответ:

$$
\begin{aligned}
p=\frac{u^{\prime}+u}{2}, & q=\frac{u^{\prime}+v}{2}, & r=u^{\prime}+l_{1}, \\
p^{\prime}=\frac{u+u^{\prime}}{2}, & q^{\prime}=\frac{u+v^{\prime}}{2}, & r^{\prime}=u+l_{1}^{\prime} .
\end{aligned}
$$

Вспоминая определение допустимых систем координат на седловых атомах без звездочек, для первого 3 -атома $C_{2}$ имеем:

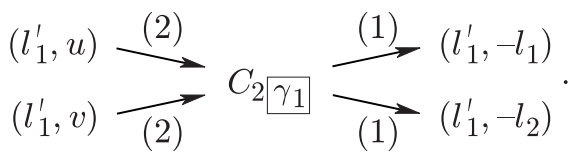

Стрелочки указывают направление роста $K_{0}$. Цифры над стрелочками обозначают семейство торов, к которому относится данный граничный тор. Знак минус требуется для согласования ориентаций систем координат на атоме. Для оставшихся дуг имеем:

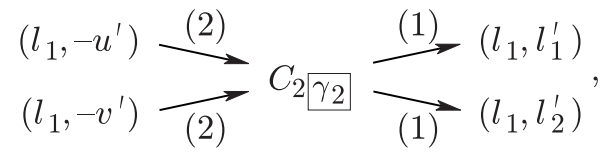

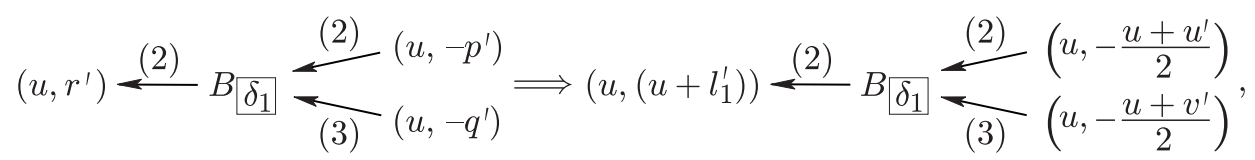

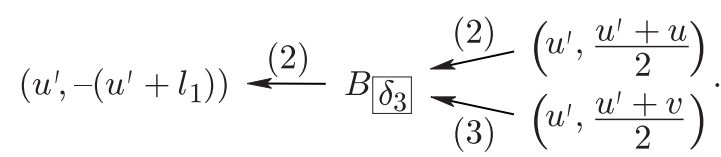

Естественно, оба атома $B$ имеют по второму экземпляру, которые отличаются заменой первого базисного цикла $u \rightarrow v$ и $u^{\prime} \rightarrow v^{\prime}$. Для краткости мы их тут не приводим.

Теперь, чтобы достичь поставленной цели, достаточно учесть, что первый базисньй цикл - это всегда однозначно определенный цикл этой бифуркации. Откуда имеем:

$$
\lambda_{\gamma_{1}}=l_{1}^{\prime}=l_{2}^{\prime}, \quad \lambda_{\gamma_{2}}=l_{1}=l_{2}, \quad \lambda_{\delta_{1}}=u=v, \quad \lambda_{\delta_{3}}=u^{\prime}=v^{\prime}
$$


ТАБЛИЦА 2. Допустимые системы координат

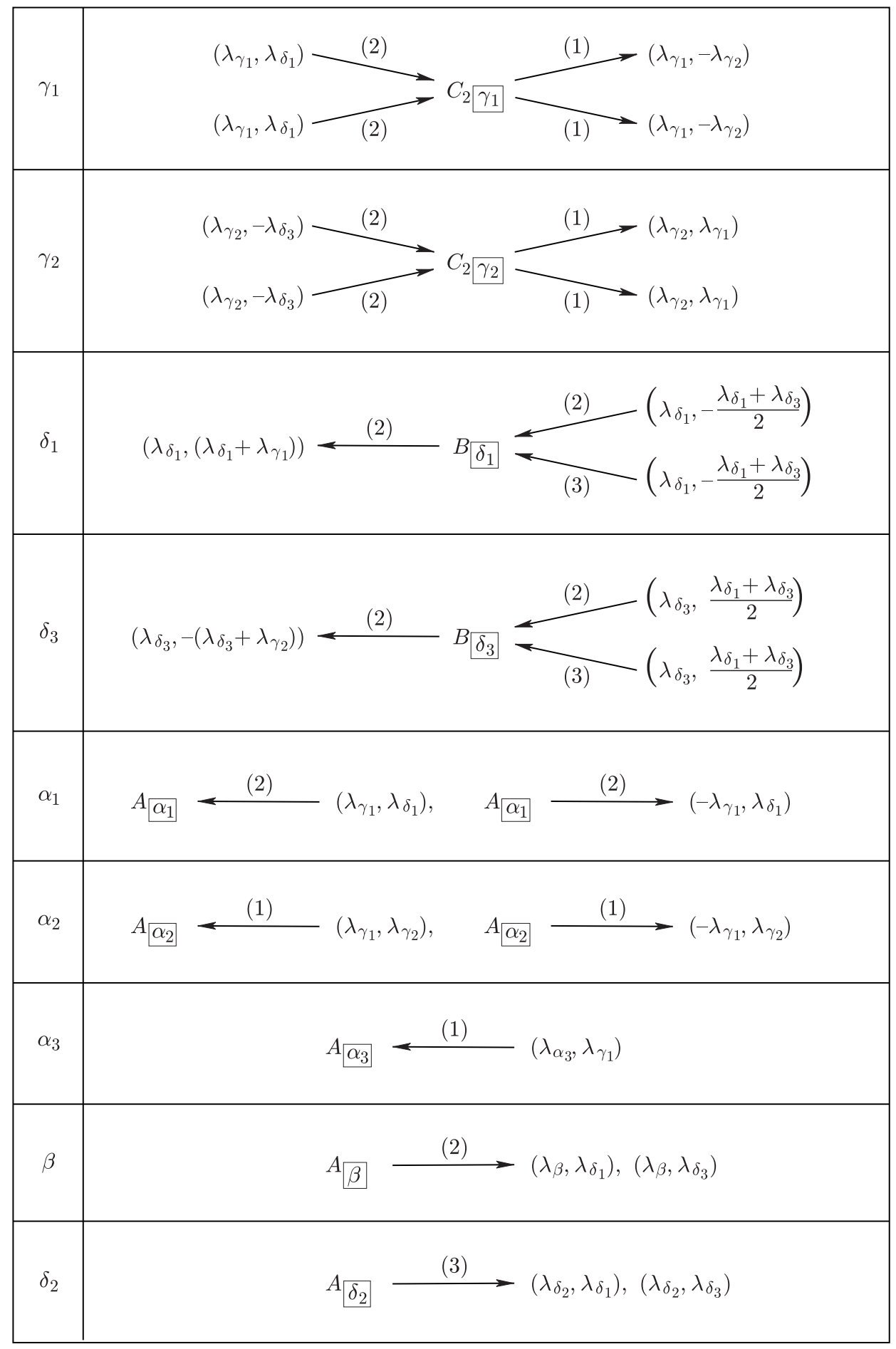


Результат представлен в таблице 2 .

Осталось выбрать системы координат на атомах $A$.

Допустимые системы координат на $A$-атомах имеют одно существенное отличие: при необходимости "отрегулировать" ориентацию меняют знак первого базисного цикла, а не второго, как в случае седловых атомов. И это понятно, так как в этом случае второй, а не первый базисньй цикл имеет естественную ориентацию, задаваемую гамильтоновьм потоком.

Вспомним понятие матрицы склейки. Рассмотрим участок допустимой кривой, содержащийся в одной камере бифуркационной диаграммы с концами на ее стенках. На торах этой кривой определены две допустимые системы координат, пришедшие с граничных торов двух бифуркаций. Ввиду нашего требования на ориентации эти два базиса будут ориентированы по-разному. Матрица, выражающая базис, пришедший сверху (по росту $f$ ), через нижний, называется матрицей склейки. Если системы координат допустимые, то это целочисленная матрица с определителем -1. Ясно, что матрица склейки несет в себе полную информацию о слоении Лиувилля в прообразе этой кривой. Формулы, выражающие метки молекулы через матрицы склеек на ее ребрах, можно найти в [12].

Выберем допустимую систему координат на $\alpha_{2}$. На $\gamma_{1}$ система координат со стороны семейства (1) уже выбрана - это $\left(\lambda_{\gamma_{1}},-\lambda_{\gamma_{2}}\right)$. Тогда пара $\left(\lambda_{\gamma_{1}}, \lambda_{\gamma_{2}}\right)$ - искомая, ибо она образует базис, удовлетворяет всем условиям допустимости и согласована с меткой $r=\infty$ на соответствующем ребре круговой молекулы особенности $N$. Действительно,

$$
\left(\begin{array}{c}
\lambda_{\gamma_{1}} \\
\lambda_{\gamma_{2}}
\end{array}\right)=\left(\begin{array}{cc}
1 & 0 \\
0 & -1
\end{array}\right)\left(\begin{array}{c}
\lambda_{\gamma_{1}} \\
-\lambda_{\gamma_{2}}
\end{array}\right) \Rightarrow r=\infty
$$

Аналогично поступаем с $\alpha_{1}$. Получаем $\left(-\lambda_{\gamma_{1}}, \lambda_{\delta_{1}}\right)$, если дугу $\alpha_{1}$ рассматривать как нижнюю, и соответственно $\left(\lambda_{\gamma_{1}}, \lambda_{\delta_{1}}\right)$, если эта дуга является верхней границей кривой.

Для дуги $\beta$ допустимую систему координат можно выбрать сразу двумя способами: это удобно сделать исходя из особенностей типа pitchfork точек $z_{1}, z_{2}$ при $g=0$. В таких случаях за второй базисный цикл берут ось смежного атома $B$ :

$$
\begin{aligned}
\left(\begin{array}{c}
\lambda_{\delta_{1}} \\
-\frac{\lambda_{\delta_{1}}+\lambda_{\delta_{3}}}{2}
\end{array}\right) & =\left(\begin{array}{ll}
0 & 1 \\
1 & *
\end{array}\right)\left(\begin{array}{c}
\lambda_{\beta} \\
\lambda_{\delta_{1}}
\end{array}\right) \Rightarrow r=0, \\
\left(\begin{array}{c}
\lambda_{\delta_{3}} \\
\frac{\lambda_{\delta_{1}}+\lambda_{\delta_{3}}}{2}
\end{array}\right) & =\left(\begin{array}{ll}
0 & 1 \\
1 & *
\end{array}\right)\left(\begin{array}{c}
\lambda_{\beta} \\
\lambda_{\delta_{3}}
\end{array}\right) \Rightarrow r=0 .
\end{aligned}
$$

Базис на дуге $\delta_{2}$ выбирается двумя способами из круговых молекул двух особенностей “бифуркация седло-узел" (точки $z_{1}$ и $z_{2}$ при $g \neq 0$ ). В этом случае также за второй базисньй цикл можно взять ось соседнего атома $B$ :

$$
\begin{aligned}
\left(\begin{array}{c}
\lambda_{\delta_{1}} \\
-\frac{\lambda_{\delta_{1}}+\lambda_{\delta_{3}}}{2}
\end{array}\right) & =\left(\begin{array}{ll}
0 & 1 \\
1 & *
\end{array}\right)\left(\begin{array}{c}
\lambda_{\delta_{2}} \\
\lambda_{\delta_{1}}
\end{array}\right) \Rightarrow r=0, \\
\left(\begin{array}{c}
\lambda_{\delta_{3}} \\
\frac{\lambda_{\delta_{1}}+\lambda_{\delta_{3}}}{2}
\end{array}\right) & =\left(\begin{array}{ll}
0 & 1 \\
1 & *
\end{array}\right)\left(\begin{array}{c}
\lambda_{\delta_{2}} \\
\lambda_{\delta_{3}}
\end{array}\right) \Rightarrow r=0 .
\end{aligned}
$$


Наконец, для дуги $\alpha_{3}$ можно выбрать допустимую систему координат исходя из особенности центр-центр точки $P$ при $g^{2}>c_{2}$ :

$$
\left(\begin{array}{c}
\lambda_{\alpha_{3}} \\
\lambda_{\gamma_{1}}
\end{array}\right)=\left(\begin{array}{cc}
* & -1 \\
-1 & 0
\end{array}\right)\left(\begin{array}{c}
-\lambda_{\gamma_{1}} \\
\lambda_{\gamma_{2}}
\end{array}\right) \Rightarrow r=0
$$

Все допустимые системы координат собраны в таблице 2.

Комментарий. Рассмотрим, к примеру, 3-атом $Q_{\gamma_{1}}$ при $g^{2}>p_{1}$. Согласно таблице 2 допустимой системой координат на нем со стороны семейства (2) является $\left(\lambda_{\gamma_{1}}, \lambda_{\delta_{1}}\right)$. При рассмотрении бифуркационной диаграммы может возникнуть предположение, что цикл $\lambda_{\delta_{1}}$ не определен. Однако это не так. Достаточно вспомнить о существовании общей трехмерной бифуркационной диаграммы. В ее масштабах рассматриваемый 3-атом может быть переведен в зону $g^{2}<p_{1}$ посредством гладкой изотопии, сохраняющей все характеристики слоений. Следовательно, и базис сохранит свойство допустимости.

\section{§6. Взаимное расположение базисных циклов на торах семейств}

Зададимся простой целью: имея два базиса на одном семействе торов, составленных из $\lambda_{*}$, научимся выписьвать матрицу перехода от первого ко второму. Иными словами, мы хотим знать взаимное расположение базисных циклов на торах семейств.

Вспомним важную лемму (см. [12], [21]). Здесь и далее будем рассматривать индексы пересечения циклов на торе с точностью до знака.

Лемма. Пусть ребро молекуль соединяет две бифуркации *и**.

1) Метка $r=\infty$ тогда и только тогда, когда индекс пересечения ииклов $\lambda_{*}$ и $\lambda_{* *}$ на торах этого семейства равен 0 (т.е. иикль гомологичнь);

2) $r=0$ тогда и только тогда, когда индекс пересечения $\lambda_{*}$ и $\lambda_{* *}$ равен 1 ;

3) $r=1 / 2$ тогда и только тогда, когда индекс пересечения $\lambda_{*} u \lambda_{* *}$ равен 2.

Полезно еше одно дополнительное соображение. Рассмотрим изоэнергетическую молекулу максимальной энергии, т.е. молекулу, соответствующую сечению бифуркационной диаграммы такой прямой $h=c$, что дальнейшее увеличение $c$ является гладкой изотопией этой прямой (см. рис. 3, прямая 5). Заметим, что слоение Лиувилля в ее прообразе одинаково при всех значениях $g$. Действительно, все эти прямые можно перевести друг в друга посредством гладкой изотопии в масштабах трехмерной бифуркационной диаграммы, обсуждавшейся ранее. Эта молекула имеет вид:

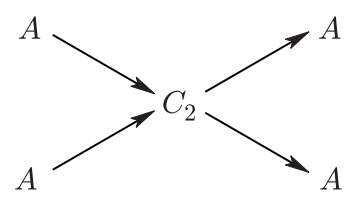

При $g^{2}>p_{2}$ из круговых молекул точек $N$ и $P$, применяя лемму в семействе $(1)$, получаем, что циклы $\lambda_{\gamma_{1}}$ и $\lambda_{\alpha_{2}}$ гомологичны, а циклы $\lambda_{\alpha_{2}}$ и $\lambda_{\alpha_{3}}$ имеют индекс пересечения 1. Следовательно, циклы $\lambda_{\alpha_{3}}$ и $\lambda_{\gamma_{1}}$ тоже имеют индекс пересечения 1. 
Аналогичные рассуждения справедливы для семейства (2). В итоге мы получаем, что все $r$-метки молекулы максимальной энергии равны нулю. В дальнейшем будем пользоваться этой молекулой наравне с круговыми.

Теперь перейдем от общих замечаний к рассмотрению каждого семейства торов по отдельности.

Семейство (1). На этом семействе нами выбраны базисы, составленные из циклов $\lambda_{\gamma_{1}}$ и $\lambda_{\gamma_{2}}$, а также базис $\left(\lambda_{\alpha_{1}}, \lambda_{\gamma_{1}}\right)$, соответствующий дуге $\alpha_{3}$. Матрицы склеек между базисами первой категории выписываются автоматически. Оказывается, что нет необходимости явно выражать через них последний базис. Действительно, для кривой, соединяющей дуги $\alpha_{3}$ и $\alpha_{2}$ бифуркационной диаграммы, можно сразу выписать меченую молекулу (таблица 4 № 1), так как она совпадает с круговой молекулой особенности центр-центр, всегда имеющей такой вид.

Лиувиллево слоение в прообразе кривой, соединяющей дуги $\alpha_{3}$ и $\gamma_{1}$, совпадает со слоением в прообразе кривой, соединяющей дуги $\gamma_{1}$ и $\alpha_{2}$ при $g^{2}<p_{1}$, так как они могут быть переведены одна в другую посредством гладкой изотопии в масштабах трехмерной бифуркационной диаграммы. Матрицу склейки, соответствующую последней кривой, мы знаем.

Наконец, если дуга $\alpha_{3}$ соединена сама с собой, то молекула имеет вид, указанньй в таблице 4 под номером 6 , как и всякая круговая молекула неособой точки $A$-дуги бифуркационной диаграммы $[12 ;$ т. 2 , гл. 1$]$.

Семейство (2). Здесь придется провести более детальный анализ. Будем изображать фундаментальную группу тора в виде целочисленной решетки на плоскости, а циклы - в виде векторов на ней.

При построении допустимых систем координат мы использовали следующие однозначно определенные циклы бифуркации, относящиеся к данному семейству: $\lambda_{\beta}, \lambda_{\gamma_{1}}, \lambda_{\gamma_{2}}, \lambda_{\delta_{1}}, \lambda_{\delta_{3}}$.

Рассматривая $r$-метки на ребрах круговых молекул и молекулы максимальной энергии, находим индексы пересечений этих циклов:

\begin{tabular}{|c|c|c|c|c|c|}
\hline & $\lambda_{\beta}$ & $\lambda_{\gamma_{1}}$ & $\lambda_{\gamma_{2}}$ & $\lambda_{\delta_{1}}$ & $\lambda_{\delta_{3}}$ \\
\hline$\lambda_{\beta}$ & 0 & 1 & 1 & 1 & 1 \\
\hline$\lambda_{\gamma_{1}}$ & 1 & 0 & & 1 & \\
\hline$\lambda_{\gamma_{2}}$ & 1 & & 0 & & 1 \\
\hline$\lambda_{\delta_{1}}$ & 1 & 1 & & 0 & 2 \\
\hline$\lambda_{\delta_{3}}$ & 1 & & 1 & 2 & 0 \\
\hline
\end{tabular}

Будем (условно) базисы, “торчащие” снизу на целочисленной решетке тора, изображать положительно ориентированными, а “торчащие” сверху - отрицательно ориентированными. Для торов семейства (2) имеем следующую информацию об ориентации базисов:

\begin{tabular}{|c|c|}
\hline+ & - \\
\hline$\left(\lambda_{\delta_{1}}, \lambda_{\delta_{1}}+\lambda_{\gamma_{1}}\right)$ & $\left(\lambda_{\gamma_{1}}, \lambda_{\delta_{1}}\right)$ \\
\hline$\left(\lambda_{\delta_{3}},-\left(\lambda_{\delta_{3}}+\lambda_{\gamma_{2}}\right)\right)$ & $\left(\lambda_{\gamma_{2}},-\lambda_{\delta_{3}}\right)$ \\
\hline$\left(\lambda_{\beta}, \lambda_{\delta_{1}}\right)$ & $\left(\lambda_{\delta_{1}},-\frac{\lambda_{\delta_{1}}+\lambda_{\delta_{3}}}{2}\right)$ \\
\hline$\left(\lambda_{\beta}, \lambda_{\delta_{3}}\right)$ & $\left(\lambda_{\delta_{3}}, \frac{\lambda_{\delta_{1}}+\lambda_{\delta_{3}}}{2}\right)$ \\
\hline
\end{tabular}


Первый базис $\left(\lambda_{\gamma_{1}}, \lambda_{\delta_{1}}\right)$ изображаем произвольно, так как нам важно взаимное расположение циклов (см. рис. 5). Далее, посредством несложного перебора убеждаемся, что перечисленные выше условия однозначно определяют положение оставшихся циклов. Результат изображен на рис. 5.

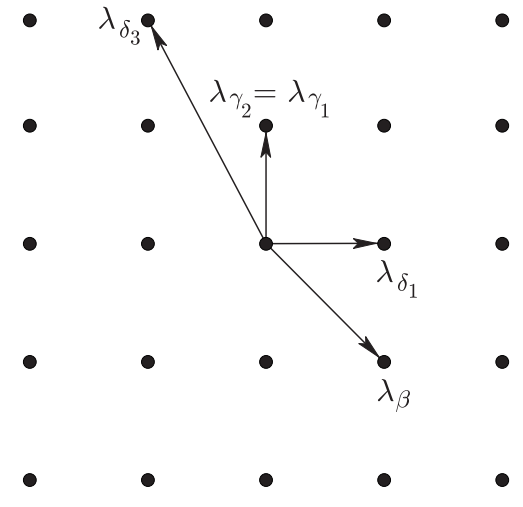

Семейство (2)

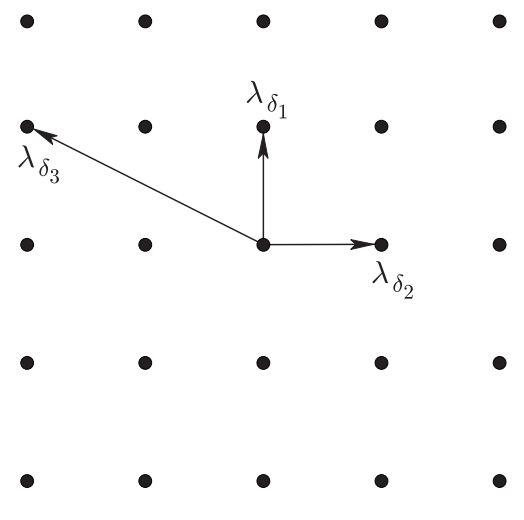

Семейство (3)

Рис. 5.

Семейство (3). Однозначно определенные циклы бифуркации, относящиеся к данному семейству, таковы: $\lambda_{\delta_{1}}, \lambda_{\delta_{2}}, \lambda_{\delta_{3}}$.

Из круговых молекул имеем следуюшую информацию об индексах пересечения:

\begin{tabular}{|c|c|c|c|}
\hline & $\lambda_{\delta_{1}}$ & $\lambda_{\delta_{2}}$ & $\lambda_{\delta_{3}}$ \\
\hline$\lambda_{\delta_{1}}$ & 0 & 1 & 2 \\
\hline$\lambda_{\delta_{2}}$ & 1 & 0 & 1 \\
\hline$\lambda_{\delta_{3}}$ & 2 & 1 & 0 \\
\hline
\end{tabular}

Для торов семейства (3) имеем следующую информацию об ориентации базисов:

\begin{tabular}{|c|c|}
\hline+ & - \\
\hline$\left(\lambda_{\delta_{2}}, \lambda_{\delta_{1}}\right)$ & $\left(\lambda_{\delta_{1}},-\frac{\lambda_{\delta_{1}}+\lambda_{\delta_{3}}}{2}\right)$ \\
\hline$\left(\lambda_{\delta_{2}}, \lambda_{\delta_{3}}\right)$ & $\left(\lambda_{\delta_{3}}, \frac{\lambda_{\delta_{1}}+\lambda_{\delta_{3}}}{2}\right)$ \\
\hline
\end{tabular}

Результат представлен на рис. 5 .

Рисунок 5 следует считать ответом к поставленной в настоящей работе задаче. Действительно, зная взаимное расположение базисных циклов на торах и все допустимые системы координат, можно вычислить молекулу, соответствующую любой допустимой кривой. Несколько иначе обстоит дело с семейством (1). Для торов этого семейства мы не указали картинки на рис. 5. Это связано с тем, что в камере I присутствует особенность типа фокус-фокус. Обход вокруг этой особой точки дает нетривиальную матрицу монодромии. Поэтому матрица склейки на ребре молекулы зависит не только от бифуркаций, которые она соединяет, но и от 
пути, по которому проходит соответствующая допустимая кривая. Из-за этого однозначно указать взаимное расположение базисных циклов на торах семейства (1) невозможно. Впрочем, метки на этих ребрах несложно вычисляются при помощи дополнительных рассуждений, которые уже излагались выше.

Итак, для вычисления меченой молекулы, соответствующей произвольной допустимой кривой, следует действовать таким образом:

1) изобразить кривую на бифуркационной диаграмме;

2) задать на кривой ориентацию, т.е. выбрать направление роста $f$;

3) выписать допустимые системы координат на атомах, лежащих в прообразе кривой; подкорректировать ориентации атомов, согласуя их с ориентацией на кривой. Напомним, что при смене ориентации на седловых атомах меняются знаки всех вторых базисных циклов, а в случае атома $A$ - знак первого базисного цикла;

4) выписать матрицы склеек на ребрах молекулы, пользуясь рис. 5 (кроме ребер, относящихся к семейству (1));

5) в соответствии с формулами, приведенными в [12; т. 1, гл. 4], вычислить по матрицам склеек числовые метки $r, \varepsilon$ и $n$.

\section{§7. Классификация слоений Лиувилля на изоэнергетических поверхностях систем случая Клебша}

В качестве примера рассмотрим важный случай изоэнергетических молекул. Как уже отмечалось, эти молекулы соответствуют прямым $\alpha h+\beta k=c$, изображенным на рис. 3 . Будем ориентировать их по возрастанию $K_{0}$.

Номерам прямых с рис. 3 соответствуют молекулы в таблицах 3 и 4 . В таблице 3 собраны молекулы с матрицами склеек. Меченые молекулы, получающиеся в результате, собраны в таблице 4. Молекулы 10 и 12 совпадают с точностью до ориентации. В этой таблице также указаны топологические типы изоэнергетических поверхностей, вычисленные А.А. Ошемковьг [14], [15]. Через $N^{3}$ обозначена связная сумма $\left(S^{1} \times S^{2}\right) \#\left(S^{1} \times S^{2}\right) \#\left(S^{1} \times S^{2}\right)$. Итак, нами доказана следующая теорема, целиком описывающая слоения Лиувилля на трехмерных изоэнергетических поверхностях в случае Клебша.

ТЕОРема 2. В случае Клебиа обнаружсивается ровно десять различных изоэнергетических молекул. Их полный список приведен таблице 4.

\section{§ 8. Следствия: сравнение со случаем интегрируемости Эйлера}

Напомним, пожалуй, самый важный случай интегрируемости движения тяжелого твердого тела - случай Эйлера. Он получается при рассмотрении твердого тела, закрепленного в центре масс, в однородном поле сил (поле тяжести). В случае Эйлера гамильтониан и дополнительньй интеграл вьплядят так:

$$
\begin{aligned}
H & =\frac{S_{1}^{2}}{2 A_{1}}+\frac{S_{2}^{2}}{2 A_{2}}+\frac{S_{1}^{3}}{2 A_{3}}, \\
K & =S_{1}^{2}+S_{2}^{2}+S_{3}^{2} .
\end{aligned}
$$




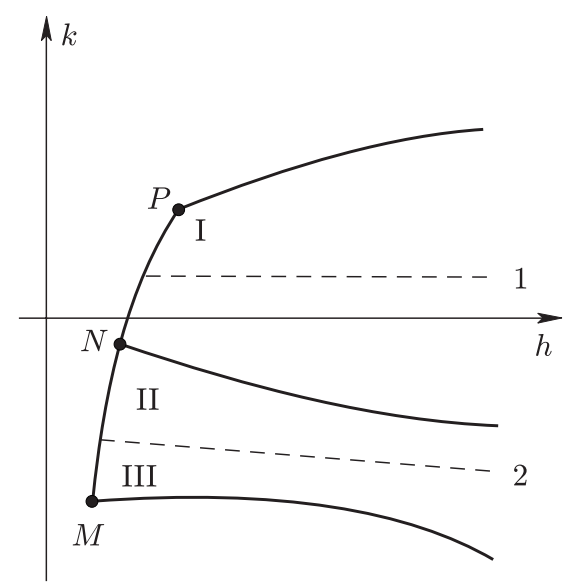

Случай Клебша

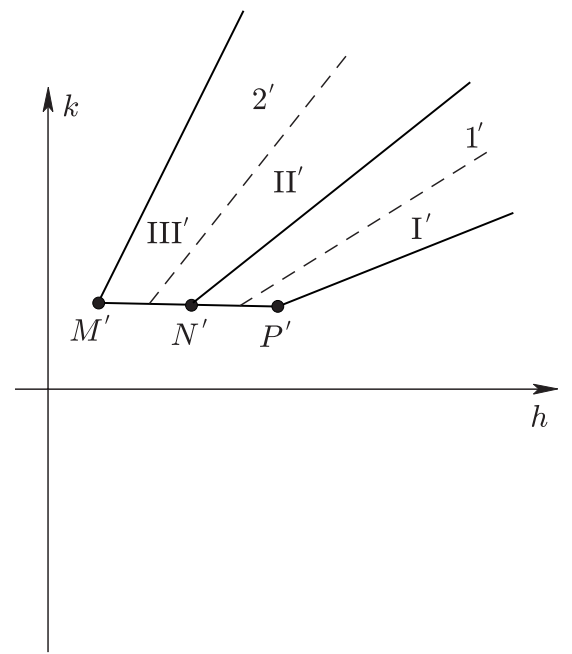

Случай Эйлера

a)

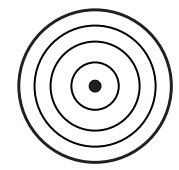

$D_{1}$

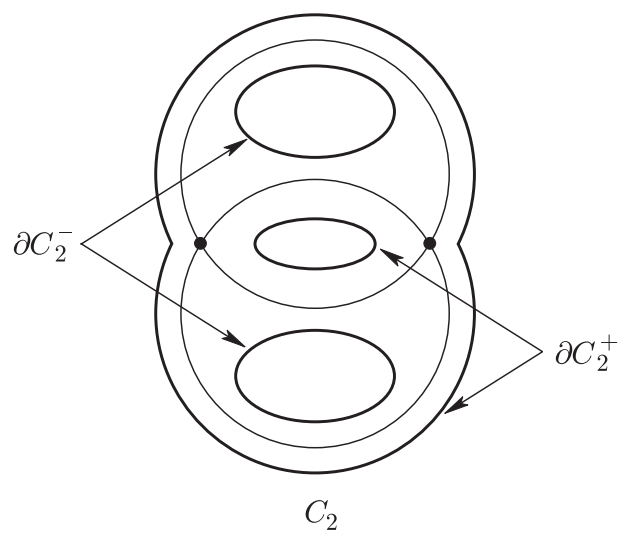

b)

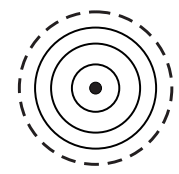

$D_{2}$

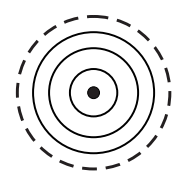

$D_{3}$

Рис. 6.

Знание инвариантов Фоменко-Цишанга позволяет сравнивать топологию лиувиллева слоения фазового пространства гамильтоновых систем, возникающих из разных физических задач. Так, например, в работе А. В. Болсинова и А. Т. Фоменко [24] установлена траекторная эквивалентность случая интегрируемости Эйле- 
ТАБлицА 3. Матрицы склеек изоэнергетических молекул

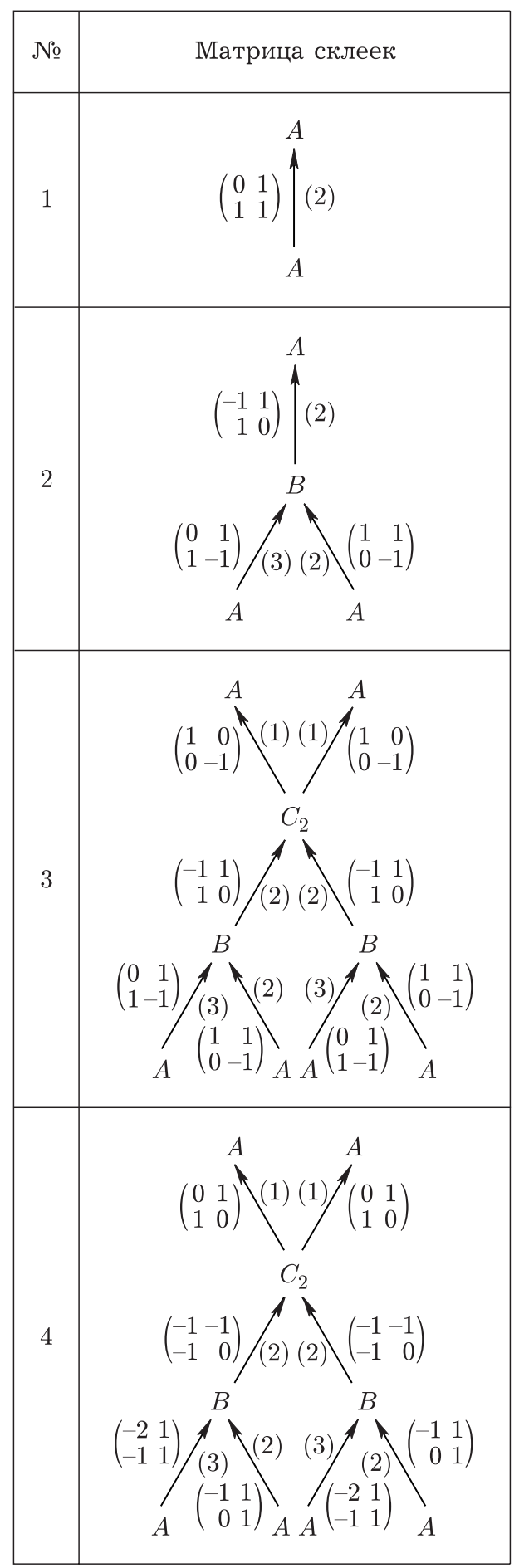

\begin{tabular}{|c|c|}
\hline № & Матрица склеек \\
\hline 5 & 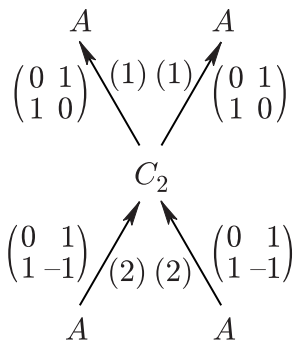 \\
\hline 6 & $\left.(2)\right|_{A} ^{A}\left(\begin{array}{rr}-1 & 0 \\
0 & 1\end{array}\right)$ \\
\hline 7 & 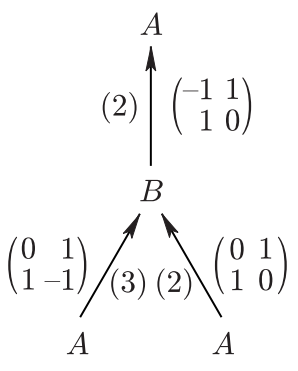 \\
\hline 8 & 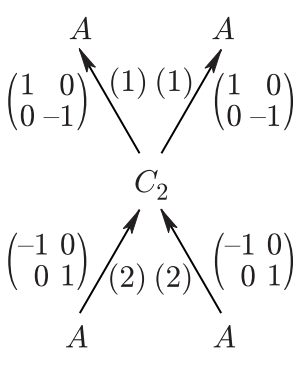 \\
\hline
\end{tabular}


ТАБлицА 3 (продолжение)

\begin{tabular}{|c|c|}
\hline № & Матрица склеек \\
\hline 9 & 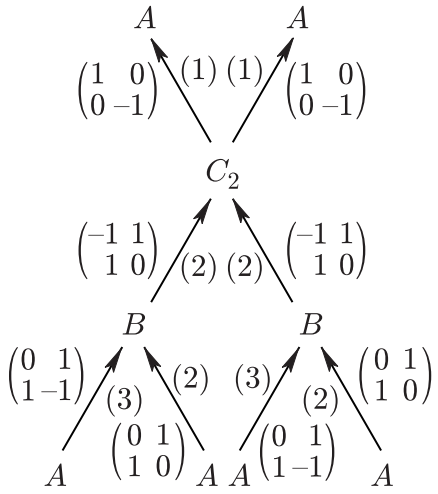 \\
\hline 10 & $\begin{array}{c}\left(\begin{array}{rr}1 & 0 \\
0 & -1\end{array}\right) \overbrace{A}^{A}(1)(1) /\left(\begin{array}{ll}1 & 0 \\
0 & -1\end{array}\right) \\
\left(\begin{array}{ll}0 & 1 \\
1 & 1\end{array}\right) /(2)(2)>\left(\begin{array}{ll}0 & 1 \\
1 & 1\end{array}\right) \\
A\end{array}$ \\
\hline
\end{tabular}

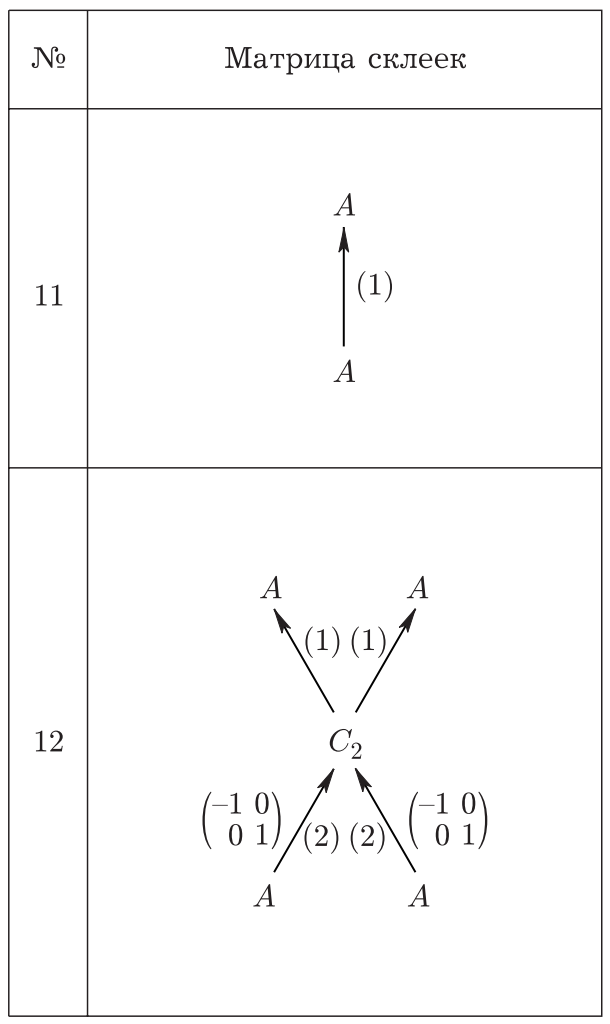

ра в динамике твердого тела и задачи Якоби о геодезических на эллипсоиде. В [12; т. 2, гл. 6] также доказана траекторная эквивалентность систем Эйлера и Клебша на трехмерных изоэнергетических поверхностях при достаточно больших значениях энергии $H=h$ и при дополнительном условии $g=0$, откуда, в частности, следует и их лиувиллева эквивалентность на этих поверхностях. Проведенное нами исследование дает новый способ доказательства этого факта, при этом удается отказаться от ограничения $g=0$ (п. 1 теоремы 3). В п. 2 теоремы 3 сформулирован новый результат, который также удается получить на основании проведенных нами исследований.

\section{Teopema 3.}

1. При достаточно больших значениях энергии случаи Эйлера и Клебша лиувиллево әквивалентны.

2. Случай Клебша при $g^{2}>p_{2}$ лиувиллево әквивалентен случаю Эйлера с ненулевым значением постоянной площадей.

ДокАЗАТЕЛьство. Инварианты Фоменко-Цишанга, а также информацию о типах особых точек случая Эйлера можно найти в [12]. Первое утверждение теоремы следует из сопоставления молекул максимальной энергии. Они изоморфны.

Во втором пункте теоремы утверждается сушествование послойного гомеомор- 
ТАБлицА 4. Изоэнергетические молекулы

\begin{tabular}{|c|c|}
\hline № & Молекула (изоэнергетическая) \\
\hline 1 & 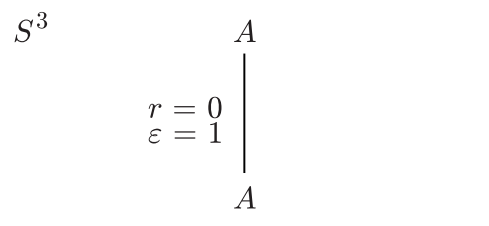 \\
\hline 2 & 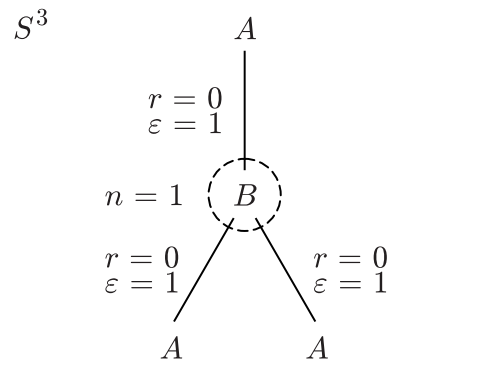 \\
\hline 3 & 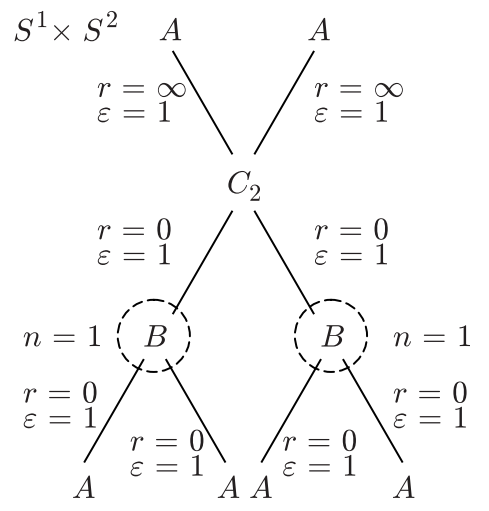 \\
\hline 4 & 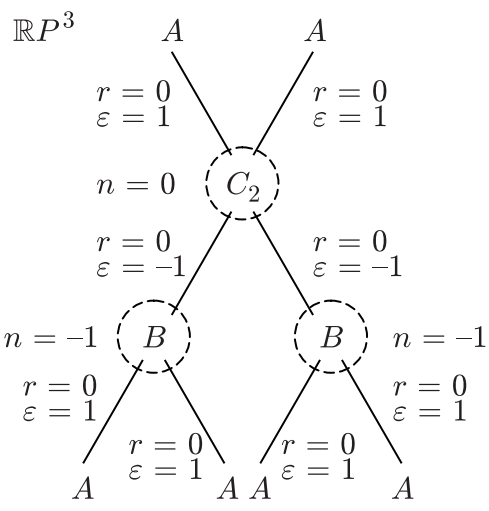 \\
\hline
\end{tabular}

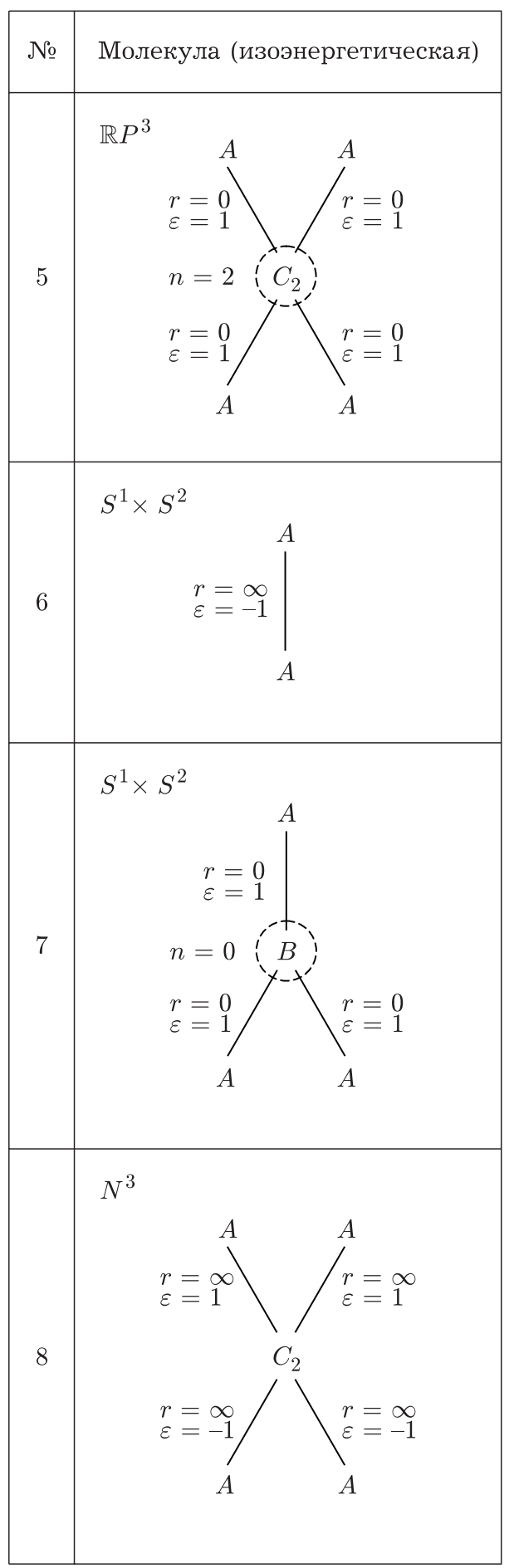


ТАБлицА 4 (продолжение)

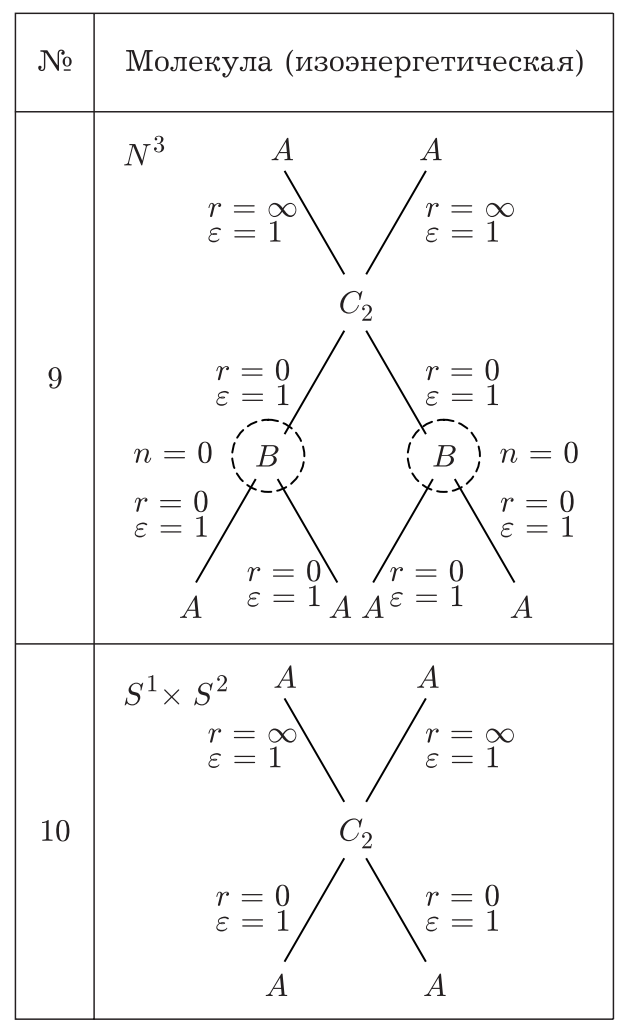

\begin{tabular}{|c|c|}
\hline № & Молекула (изоэнергетическая) \\
\hline 11 & $\begin{array}{l}S^{3} \\
\left.\quad \begin{array}{l}r=0 \\
\varepsilon=1\end{array}\right|_{A} ^{A}\end{array}$ \\
\hline 12 & 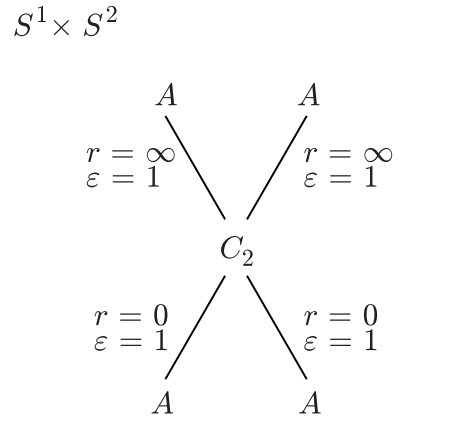 \\
\hline
\end{tabular}

физма четырехмерных поверхностей

$$
\varphi: M_{g}^{4} \rightarrow M_{g^{\prime}}^{\prime 4}
$$

Здесь поверхность $M$ соответствует случаю Клебша, а $M^{\prime}$ - случаю Эйлера; $g^{2}>p_{2}$ и $g^{\prime} \neq 0$.

Бифуркационные диаграммы двух случаев для этих значений постоянной площадей $g$ и $g^{\prime}$ изображены на рис. 6а). Рассмотрим разрезы $1,2,1^{\prime}$ и $2^{\prime}$, разделяющие бифуркационные диаграммы на зоны, обозначенные римскими цифрами.

Прообраз каждой из зон I, I' , III и III' состоит из объединения двух одинаковых окрестностей особенности типа центр-центр. На рис. 6b) изображены замкнутый диск $D_{1}$ и открытый диск $D_{2}$, каждый из которых расслоен на окружности. Известно, что любая окрестность особенности центр-центр со структурой слоения Лиувилля может быть задана как $D_{1} \times D_{2}$ (см. [12; т. 1, гл. 9]). Краем многообразия служит расслоенное на торы Лиувилля полноторие $\partial D_{1} \times D_{2}$.

Прообразы зон II и II' являются четырехмерными окрестностями особенности седло-центр типа $A \times C_{2}$ и аналогичным образом могут быть представлены в виде прямого произведения открытого диска $D_{3}$ и двумерной поверхности $C_{2}$ с краем $\partial C_{2}$ из четырех окружностей. Две из них соответствуют разрезу 1 и образуют часть края $\partial C_{2}^{+}$, а две других - разрезу 2 , их обозначим через $\partial C_{2}^{-}$. Краем четырехмерного многообразия служит $\partial C_{2} \times D_{3}=\left(\partial C_{2}^{+} \times D_{3}\right) \cup\left(\partial C_{2}^{-} \times D_{3}\right)-$ объединение четырех расслоенных на торы Лиувилля полноторий. 
Многообразия $M_{g}^{4}$ и $M_{g^{\prime}}^{\prime 4}$ можно получить, приклеивая к "базе" $C_{2} \times D_{3}$ "ручки" вида $D_{1} \times D_{2}$ с сохранением структуры слоения Лиувилля. Для этого требуется указать послойный гомеоморфизм края “базы” и края "ручки”. В рассматриваемом случае любой из возможных способов выбора пары склеиваемых торов не окажет влияния на результат. (Так как любая допустимая замена пар склеиваемых торов эквивалентна замене “ручки” на послойно гомеоморфную ей же.) А способ склейки каждой пары торов целиком определяется метками на соответствующем ребре изоэнергетической молекулы. Поэтому в нашем случае склеивающий гомеоморфизм однозначно определяется по изоэнергетической молекуле, которые в случаях Клебша и Эйлера одинаковы. Следовательно, многообразия $M_{g}^{4}$ и $M_{g^{\prime}}^{\prime 4}$, получающиеся в результате таких склеек, будут послойно гомеоморфны. Теорема доказана.

Автор благодарит А.Т. Фоменко и А.А. Ошемкова за постоянное внимание к работе и ценные консультации.

\section{Список литературы}

1. Kirchhoff G. R. Über die Bewegung eines Rotationskörpers in einer Flüssigkeit // Crelle. 1869. V. 71. P. 237-262.

2. Clebsch A. Über die Bewegung eines Körpers in einer Flüssigkeit // Math. Ann. 1871. V. 3. P. 238-262.

3. Стеклов В. А. О движении тяжелого твердого тела в жидкости. Харьков, 1983.

4. Ляпунов $A . M$. Новый случай интегрируемости уравнений движения твердого тела в жидкости // Собрание сочинений. Т. 1. М.: Изд-во АН СССР, 1954. С. 320-324.

5. Чапльгин С. А. О некоторых случаях движения твердого тела в жидкости. Статьи 1,2 // Полное собрание сочинений. Т. 1. Л.: Изд-во АН СССР, 1933. С. 3-132.

6. Чаплыгин C.A. Новое частное решение задачи о движении твердого тела в жидкости // Полное собрание сочинений. Т. 1. Л.: Изд-во АН СССР, 1933. С. 133-152.

7. Харламова E. И. О движении твердого тела вокруг неподвижной точки в центральном ньютоновском поле сил // Изв. СО АН СССР. 1959. №6. С. 7-17.

8. Погосян Т.И., Харламов М. П. Бифуркационное множество и интегральные многообразия задачи о движении твердого тела в линейном поле сил // ПММ. 1979. Т. 43. C. $419-428$.

9. Харламов М. П. Фазовая топология одного интегрируемого случая движения твердого тела // Механика твердого тела. № 11. Киев: Наукова думка, 1979. С. 50-63.

10. Погосян Т. И. Построение бифуркационных множеств в одной задаче динамики твердого тела // Механика твердого тела. № 12. Киев: Наукова думка, 1980. С. 3-23.

11. Погосян Т. И. Области возможности движения в задаче Клебша. Критический случай // Механика твердого тела. № 16. Киев: Наукова думка, 1983. С. 19-24.

12. Болсинов A.B., Фоменко A. T. Интегрируемые гамильтоновы системы. Геометрия. Топология. Классифоикация. Т. 1, 2. Ижевск: Издательский дом "Удмуртский университет", 1999.

13. Болсинов A. В., Фоменко A. T. Введение в топологию интегрируемых гамилтоновых систем. М.: Наука, 1997.

14. Ошемков $A$. A. Описание изоэнергетических поверхностей интегрируемых гамильтоновых систем с двумя степенями свободы // Труды семинара по векторному и тензорному анализу. № 23. М.: МГУ, 1988. С. 122-132.

15. Ошемков A.A. Вычисление инвариантов Фоменко для основных интегрируемых случаев динамики твердого тела // Труды семинара по векторному и тензорному анализу. № 25, часть 2. М.: МГУ, 1993. С. 23-109.

16. Ламб Г. Гидродинамика. М.-Л.: ГИТТЛ, 1947.

17. Магнус К. Гироскоп. Теория и применение. М.: Наука, 1973. 
18. Козлов В. В. Симметрии, топология и резонансы в гамильтоновой механике. Ижевск: Изд-во УдГУ, 1995.

19. Новиков С. П., Шмельцер И. Периодические решения уравнений Кирхгофа свободного движения твердого тела в идеальной несжимаемой жидкости и расширенная теория Люстерика-Шнирелмана-Морса (ЛМШ) // Функц. анализ и его прилож. 1982. Т. 15. №3. C. 54-66.

20. Харламов М. П. Топологический анализ интегрируемых задач в динамике твердого тела. Л.: Изд-во ЛГУ, 1988.

21. Болсинов А.В., Рихтер П., Фоменко А.Т. Метод круговых молекул и топология волчка Ковалевской // Матем. сб. 2000. Т. 191. № 2. С. 3-42.

22. Калашников В.В. (мл.). Об устойчивости топологической структуры боттовских интегрируемых гамилтоновых систем с двумя степенями свободы // [12]. Т. 2, приложение 2.

23. Калашников В. В. (мл.). Типичные интегрируемые гамильтоновы системы на четырехмерном симплектическом многообразии // Изв. РАН. Сер. матем. 1998. Т. 62. № 2. C. $49-74$.

24. Болсинов A. B., Фоменко A. T. Геодезический поток эллипсоида траекторно эквивалентен интегрируемому случаю Эйлера в динамике твердого тела // Докл. РАН. 1994. T. 339. № 3. C. 293-296.

Московский государственнњй

Поступила в редакцию

университет им. М.В. Ломоносова

31.10 .2001 